\title{
Uniqueness of minimal morphisms of logarithmic schemes
}

\author{
Jonathan Wise
}

\begin{abstract}
We give a sufficient condition under which the moduli space of morphisms between logarithmic schemes is quasifinite over the moduli space of morphisms between the underlying schemes. This implies that the moduli space of stable maps from logarithmic curves to a target logarithmic scheme is finite over the moduli space of stable maps, and therefore that it has a projective coarse moduli space when the target is projective.
\end{abstract}

\section{Introduction}

Chen [Che14], Abramovich and Chen [AC14], and Gross and Siebert [GS13] have recently constructed a moduli space of stable maps from logarithmic curves into logarithmic target schemes. In [Wis16], we extended the existence results of those papers beyond logarithmic curves and eliminated some technical restrictions that applied even to curves. However, [Wis16] only asserts that the moduli space of maps between logarithmic schemes exists as an algebraic space that is locally of finite presentation; Chen showed that when the target has a rank 1 logarithmic structure, the moduli space of logarithmic maps is a disjoint union of pieces, each of which is finite over the moduli space of maps between the underlying schemes [Che14, Proposition 3.7.5]. This implies in particular that the moduli space of stable maps from logarithmic curves has a projective coarse moduli space when the target variety is projective. That result was extended to so-called generalized Deligne-Faltings logarithmic structures by Abramovich and Chen [AC14].

We will explain these results with a general criterion on the domain that applies to arbitrary logarithmic targets.

Theorem 1.1. Let $S$ be a fine logarithmic scheme. Let $X$ and $Y$ be fine logarithmic $S$-schemes, with $X$ also geometrically reduced, proper, and integral (in the logarithmic sense [Kat89, Definition (4.3)]) over $S$. If the relative characteristic monoid of $X / S$ is generically trivial, then the projection from the space of logarithmic maps to the space of maps of underlying schemes factors as a monomorphism followed by an étale map

$$
\underline{\operatorname{Hom}}_{\mathbf{L o g S c h} / S}(X, Y) \rightarrow \mathscr{T} \rightarrow \operatorname{Hom}_{\mathrm{Sch} / \underline{S}}(\underline{X}, \underline{Y}),
$$

where $\mathscr{T}$ is the space of types.

The space of types is defined in Definition 3.1.

Received 15 May 2017, accepted in final form 15 September 2017.

2010 Mathematics Subject Classification 14D20, 14H10, 14D22.

Keywords: logarithmic geometry, moduli spaces, curves, stable maps.

This journal is (c) Foundation Compositio Mathematica 2019. This article is distributed with Open Access under the terms of the Creative Commons Attribution Non-Commercial License, which permits non-commercial reuse, distribution, and reproduction in any medium, provided that the original work is properly cited. For commercial re-use, please contact the Foundation Compositio Mathematica.

Supported by an NSA Young Investigator's Grant, Award \#H98230-14-1-0107. 


\section{UNIQUENESS OF MINIMAL MORPHISMS}

The following corollary recovers and generalizes the results of Chen, Abramovich-Chen, and Gross-Siebert mentioned earlier.

Corollary 1.2. Let $Y$ be a fine, saturated logarithmic scheme over $S$. Fix a type $u$ (see Definition 3.1). Let $M_{u}(Y)$ be the moduli space of stable maps from logarithmic curves to $Y$ of type $u$, let $\underline{M}_{u}(Y)$ be its underlying algebraic stack, and let $M(\underline{Y})$ be the moduli space of stable maps to $Y$. Then $\underline{M}_{u}(Y)$ is finite over $M(\underline{Y})$.

Proof. It has been shown elsewhere that the moduli space of stable logarithmic maps is locally of finite type [Wis16, Theorem 1.1], bounded [ACMW17, Proposition 1.5.6], and satisfies the valuative criterion for properness [ACMW17, Proposition 1.4.3] over stable maps, so it remains only to show that the geometric fibers are finite.

It is therefore sufficient to consider a base $S$ whose underlying scheme is the spectrum of an algebraically closed field and fix a logarithmic curve $X$ over $S$. This corresponds to a map from $S$ to the moduli space of curves, and we wish to show that the base change $M_{u, X}(Y)$ of $M_{u}(Y)$ via this map is finite over the base change $M_{u, X}(\underline{Y})$ of $M_{u}(\underline{Y})$. The space $M_{u, X}(Y)$ is the saturation ${ }^{1}$ of the logarithmic algebraic space $\underline{\operatorname{Hom}}_{\mathbf{L o g S c h} / S}(X, Y)_{u}$ obtained from $\underline{\operatorname{Hom}}_{\mathbf{L o g S c h} / S}(X, Y)$ by base change via $u: S \rightarrow \mathscr{T}$. Therefore, the projection from $M_{u, X}(Y)$ to $S$ can be factored:

$$
M_{u, X}(Y) \rightarrow \underline{\operatorname{Hom}}_{\mathbf{L o g S c h} / S}(X, Y)_{u} \rightarrow S .
$$

The first map is the saturation, hence is quasifinite; the second is geometrically injective by Theorem 1.1.

\section{Conventions and notation}

We have retained the notation of [Wis16] as much as seemed practical. In particular, $\underline{X}$ is the underlying space or stack of a logarithmic algebraic space or stack $X$. We have consistently used underlined Roman characters to represent schemes, even when no logarithmic scheme is present for the schemes to underlie, but we have not applied the same convention to morphisms of schemes, lest the underlines overwhelm.

We have made one departure from the notation of [Wis16], however. In order to distinguish pullback of logarithmic structures from pullback of étale sheaves, we apply a superscript $*$ to a morphism of schemes for the former and a superscript -1 for the latter. In [Wis16], a superscript $*$ was used for both. The superscript $*$ is sometimes also applied to a morphism of $\log a-$ rithmic schemes, and we direct the reader to Section 5 for an explanation of that usage.

When $A$ and $B$ are objects that vary with objects of some category $\mathscr{C}$, we write $\operatorname{Hom}_{\mathscr{C}}(A, B)$ for the functor or fibered category on $\mathscr{C}$ of morphisms from $A$ to $B$. This convention conflicts with the more standard convention of using the subscript to indicate in which category the homomorphisms should be taken. Our perspective is that Hom should only be applied to pairs of objects of the same type, and that it should be possible to infer the type from the arguments.

\section{Outline of the argument}

The fiber of (1.1) over a fixed type $u \in \mathscr{T}$ is the collection of minimal logarithmic maps of that type. Our demonstration that there are only finitely many minimal maps proceeds by showing

\footnotetext{
${ }^{1}$ The reason for the saturation is a matter of conventions in the literature. The space of morphisms $\underline{\operatorname{Hom}}_{\operatorname{LogSch} / S}(X, Y)$ was constructed in the category of fine, but not necessarily saturated, logarithmic structures [Wis16], whereas the moduli space of stable maps from logarithmic curves to a logarithmic target is usually understood to be defined only over fine, saturated, logarithmic schemes [GS13]. To pass from the one to the other, one must saturate.
} 


\section{J. WISE}

first that, provided that the relative characteristic of $X / S$ is generically trivial, the characteristic monoid of a minimal logarithmic map is determined by the type (Corollary 6.2). Once the characteristic monoid is determined, Lemma 6.3 implies that the collection of minimal objects is either empty or a torsor under an explicitly determined group. We show in Section 7 that this group is trivial, which completes the proof.

\section{A preliminary reduction}

In order to simplify the notation, we make a preliminary reduction. To prove Theorem 1.1, it is sufficient to work relative to $\operatorname{Hom}_{\mathbf{S c h} / \underline{S}}(\underline{X}, \underline{Y})$ and assume that an $\underline{S}$-morphism $f: \underline{X} \rightarrow \underline{Y}$ has already been fixed. Then we have an equivalence of categories:

$$
\operatorname{Hom}_{\mathbf{L o g S c h} / S}(X, Y)_{f}=\operatorname{Hom}_{\mathbf{L o g S c h} / S}\left(f^{*} M_{Y}, M_{X}\right) .
$$

On the left side, Hom should be interpreted as morphisms of logarithmic schemes over $S$ with fixed morphism of underlying schemes $f$; on the right side, Hom refers to morphisms of logarithmic structures commuting with the structural maps from $\pi^{*} M_{S}$.

Equation (2.1) informs us that we can dispense with $Y$ and work entirely on $X$, setting $M=f^{*} M_{Y}$. The following assumptions will remain in force for the rest of the paper:

(i) $S=\left(\underline{S}, M_{S}\right)$ and $X=\left(\underline{X}, M_{X}\right)$ are fine logarithmic schemes and $\pi: X \rightarrow S$ is a morphism of logarithmic schemes;

(ii) $M$ is a fine logarithmic structure on $X$, equipped with a homomorphism of logarithmic structures $\pi^{*} M_{S} \rightarrow M$.

Theorem 1.1 breaks up into the following two statements. For the space of types mentioned in the first, see Definition 3.1.

Theorem 2.1. Assume that $\underline{X}$ is proper over $\underline{S}$. Then the space of types is étale over $\underline{S}$.

For the next statement, we introduce some notation. For any type $u$, denote the space of morphisms of type $u$ by $\operatorname{Hom}_{\mathbf{L o g S c h} / S}\left(M, M_{X}\right)_{u}$.

Theorem 2.2. Assume that $\underline{S}$ is the spectrum of an algebraically closed field, that $\pi^{*} M_{S} \rightarrow M_{X}$ is an integral homomorphism of monoids, that $\underline{X}$ is reduced, and that $\underline{X}$ is proper over $\underline{S}$. If the sheaf of relative characteristic monoids $M_{X} / \pi^{*} M_{S}$ vanishes on a dense open subset of $X$, then for any type $u$, the underlying algebraic space of $\operatorname{Hom}_{\mathbf{L o g S c h} / S}\left(M, M_{X}\right)_{u}$ has at most one point.

The first of these statements is treated in Section 3, and the other takes up the balance of the paper.

\section{Types}

Definition 3.1. Let the notation be as in Section 2: $M$ is a logarithmic structure on $X$ that is integral over $M_{S}$. A type consists of a homomorphism of sheaves of abelian groups

$$
u: M^{\mathrm{gp}} / \pi^{*} M_{S}^{\mathrm{gp}} \rightarrow M_{X}^{\mathrm{gp}} / \pi^{*} M_{S}^{\mathrm{gp}} .
$$

We define

$$
\begin{aligned}
\mathscr{T} & =\operatorname{Hom}_{\mathrm{Sch} / \underline{S}}\left(M^{\mathrm{gp}} / \pi^{*} M_{S}^{\mathrm{gp}}, M_{X}^{\mathrm{gp}} / \pi^{*} M_{S}^{\mathrm{gp}}\right) \\
& =\operatorname{Hom}_{\mathrm{Sch} / \underline{S}}\left(\bar{M}^{\mathrm{gp}} / \pi^{-1} \bar{M}_{S}^{\mathrm{gp}}, \bar{M}_{X}^{\mathrm{gp}} / \pi^{-1} \bar{M}_{S}^{\mathrm{gp}}\right) .
\end{aligned}
$$




\section{UNIQUENESS OF MINIMAL MORPHISMS}

More explicitly, for any $\underline{S}$-scheme $\underline{S}^{\prime}$, write $\underline{X}^{\prime}=\underline{X} \times \underline{S} \underline{S}^{\prime}$ and $g: \underline{X}^{\prime} \rightarrow \underline{X}$ for the projection; then

$$
\begin{aligned}
\mathscr{T}\left(S^{\prime}\right) & =\operatorname{Hom}\left(g^{-1}\left(M^{\mathrm{gp}} / \pi^{*} M_{S}^{\mathrm{gp}}\right), g^{-1}\left(M_{X}^{\mathrm{gp}} / \pi^{*} M_{S}^{\mathrm{gp}}\right)\right) \\
& =\operatorname{Hom}\left(g^{-1}\left(\bar{M}^{\mathrm{gp}} / \pi^{-1} \bar{M}_{S}^{\mathrm{gp}}\right), g^{-1}\left(\bar{M}_{X}^{\mathrm{gp}} / \pi^{-1} \bar{M}_{S}^{\mathrm{gp}}\right)\right) .
\end{aligned}
$$

Proof of Theorem 2.1. For the duration of the proof, we abbreviate $F=\bar{M}^{\mathrm{gp}} / \pi^{-1} \bar{M}_{S}^{\mathrm{gp}}$ and $G=\bar{M}_{X}^{\mathrm{gp}} / \pi^{-1} \bar{M}_{S}^{\mathrm{gp}}$. The object of the proof is to show that $\operatorname{Hom}_{\mathrm{Sch} / \underline{S}}(F, G)$ is representable by an étale algebraic space over $\underline{S}$. Since $F$ and $G$ are constructible, we have ${ }^{2}$

$$
\operatorname{Hom}_{\text {ét }\left(\underline{X}^{\prime}\right)}\left(g^{-1} F, g^{-1} G\right) \simeq g^{-1} \operatorname{Hom}_{\text {ét }(\underline{X})}(F, G)
$$

for any morphism $g: \underline{X}^{\prime} \rightarrow \underline{X}$. Let us establish (3.1): since these are sheaves, it is sufficient to verify it at the stalks, so we can assume that $\underline{X}^{\prime}=x$ is the spectrum of an algebraically closed field. Consider first the case where $F$ is freely generated by the sheaf of sections of an étale scheme $Z$ of finite presentation over $X$. In that case,

$$
\begin{aligned}
\operatorname{Hom}_{\text {ét }\left(\underline{X}^{\prime}\right)}\left(g^{-1} F, g^{-1} G\right) & =\operatorname{Hom}\left(F_{x}, G_{x}\right)=\Gamma\left(Z_{x}, G_{x}\right), \\
\operatorname{Hom}_{\text {ét( } \underline{X})}(F, G)_{x} & =\underset{U}{\lim } \Gamma\left(Z_{U}, G\right),
\end{aligned}
$$

where the direct limit is taken over étale neighborhoods of $x$. As $Z_{x}$ is finite, we can identify the direct limit with $\Gamma\left(Z_{x}, G_{x}\right)$.

For the general case of (3.1), it is sufficient to prove this assertion in an affine neighborhood of each point of $\underline{X}^{\prime}$, so we can assume that $\underline{X}$ is quasicompact and quasiseparated. Therefore, by [SGA4-3, Proposition IX.2.7], the sheaf $F$ has a finite presentation, with $F_{0}$ and $F_{1}$ both freely generated by the sheaves of sections of étale schemes of finite presentation over $\underline{X}$ :

$$
F=\underset{\lim }{\longrightarrow}\left(F_{1} \rightrightarrows F_{0}\right) .
$$

Then by the universal property of the colimit, $\operatorname{Hom}_{\text {ét }(\underline{X})}(F, G)$ can be represented as an equalizer:

$$
\operatorname{Hom}_{\text {ét }(\underline{X})}(F, G)=\lim _{\longleftarrow}\left(\operatorname{Hom}_{\text {ét }(\underline{X})}\left(F_{0}, G\right) \rightrightarrows \operatorname{Hom}_{\text {ét }(\underline{X})}\left(F_{1}, G\right)\right) \text {. }
$$

But pullback preserves finite limits, and we have already seen that (3.1) holds when $F$ is free, so we have what we need:

$$
\begin{aligned}
g^{-1} \operatorname{Hom}_{\text {ét }(\underline{X})}(F, G) & =g^{-1} \varlimsup_{\lim }\left(\operatorname{Hom}_{\text {ét }(\underline{X})}\left(F_{0}, G\right) \rightrightarrows \operatorname{Hom}_{\text {ét }(\underline{X})}\left(F_{1}, G\right)\right) \\
& =\underbrace{}_{\lim }\left(\operatorname{Hom}_{\text {ét }\left(\underline{X}^{\prime}\right)}\left(g^{-1} F_{0}, g^{-1} G\right) \rightrightarrows \operatorname{Hom}_{\text {ét }\left(\underline{X}^{\prime}\right)}\left(g^{-1} F_{1}, g^{-1} G\right)\right) \\
& =\operatorname{Hom}_{\text {ét }\left(\underline{X}^{\prime}\right)}\left(g^{-1} F, g^{-1} G\right) .
\end{aligned}
$$

Thus, the espace étalé of $\operatorname{Hom}_{\text {ét }(\underline{X})}(F, G)$ represents $\operatorname{Hom}_{\mathbf{S c h} / \underline{X}}(F, G)$.

To complete the proof of the theorem, we now observe that the espace étalé of $\pi_{*} \operatorname{Hom}_{\text {ét }(\underline{X})}(F, G)$ represents $\operatorname{Hom}_{\mathbf{S c h} / \underline{S}}(F, G)$. Indeed, for any $g: \underline{S}^{\prime} \rightarrow \underline{S}$, we have

$$
\begin{aligned}
\operatorname{Hom}_{\mathbf{S c h} / \underline{S}}(F, G)\left(\underline{S}^{\prime}\right) & =\operatorname{Hom}\left(g^{-1} F, g^{-1} G\right)=\Gamma\left(\underline{S}^{\prime}, \pi_{*} \operatorname{Hom}_{\text {ét }\left(\underline{X}^{\prime}\right)}\left(g^{-1} F, g^{-1} G\right)\right) \\
& =\Gamma\left(\underline{S}^{\prime}, \pi_{*} g^{-1} \operatorname{Hom}_{\text {ét }\left(\underline{X}^{\prime}\right)}(F, G)\right) \quad \text { by }(3.1) \\
& =\Gamma\left(\underline{S}^{\prime}, g^{-1} \pi_{*} \operatorname{Hom}_{\text {ét }(\underline{X})}(F, G)\right) \quad \text { by proper base change } \\
& \quad \text { SGA4-3, Théorème } 5.1(\mathrm{i})] .
\end{aligned}
$$

This completes the proof.

${ }^{2}$ The notation in (3.1) adheres pedantically to our convention for the subscript of Hom (see Section 1). In other settings the "sheaf Hom" on the small étale site might be denoted by Hom or $\mathscr{H}$ om. 


\section{J. WISE}

\section{The left adjoint to pullback for étale sheaves}

Throughout this section, $\pi: \underline{X} \rightarrow \underline{S}$ will be flat and locally of finite presentation, with reduced geometric fibers. It was shown in [Wis16, Theorem 4.5] that, under these conditions, the pullback functor $\pi^{-1}$ for étale sheaves has a left adjoint, $\pi_{!}$. In this section, we make some further observations about this functor.

We will use the notation $G^{\text {ét }}$ for the espace étalé of an étale sheaf $G$.

Lemma 4.1. For any étale sheaf $F$ on $\underline{X}$, the fiber of $\pi_{!} F$ over a geometric point $s$ of $S$ is $\pi_{0}\left(F_{s}^{\text {ét }}\right)$, where $F_{s}^{\text {ét }}$ is the fiber of $F^{\text {ét }}$ over $s$.

Proof. Since $\pi_{\text {! }}$ commutes with arbitrary change of base [Wis16, Corollary 4.5.1], we may assume that $\underline{S}$ is the spectrum of an algebraically closed field. Since $\pi^{-1}(G)^{\text {ét }}=G^{\text {ét }} \times \underline{S} \underline{X}$ for any étale sheaf $G$ on $\underline{S}$, we have

$$
\operatorname{Hom}\left(F, \pi^{-1} G\right)=\operatorname{Hom}\left(F^{\text {ét }}, G^{\text {ét }} \underset{\underline{S}}{\times} \underline{X}\right)=\operatorname{Hom}\left(\pi_{0}\left(F^{\text {ét }} / \underline{S}\right), G^{\text {ét }}\right) .
$$

Thus, $\pi_{0}\left(F^{\text {ét }} / \underline{S}\right)$ and $\pi_{!}(F)^{\text {ét }}$ represent the same functor, hence are isomorphic.

For the next few statements, we will use the notation $\pi_{!}^{\mathbf{N}}$ for the left adjoint to $\pi^{-1}$ in the category of integral monoids [Wis16, Proposition 4.7], because this functor does not agree with

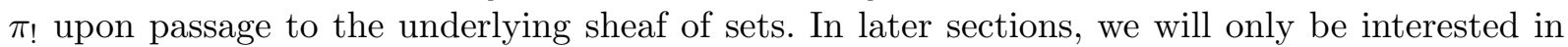
$\pi_{!}^{\mathbf{N}}$ and not in $\pi_{!}$, so we will discard the superscript from the former.

The functor $\pi^{-1}$ does commute with passage from commutative monoids to their underlying sets, so it follows formally that its left adjoint respects passage from sets to their freely generated monoids: for any sheaf of sets $F$ on $\underline{X}$, we have

$$
\pi_{!}^{\mathbf{N}}(\mathbf{N} F)=\mathbf{N} \pi_{!}(F)
$$

where we have written $\mathbf{N} F$ for the monoid freely generated by $F$.

Lemma 4.2. (i) If $F$ is an étale sheaf of integral monoids on $\underline{X}$, then $\pi_{!}^{\mathbf{N}} F$ is generated by $\pi_{!} F$.

(ii) The functor $\pi_{!}^{\mathbf{N}}$ preserves surjections.

Proof. Let $G \subset \pi_{!}^{\mathbf{N}} F$ be the submonoid generated by $\pi_{!} F$. Then $F \rightarrow \pi^{-1} \pi_{!}^{\mathbf{N}} F$ factors:

$$
F \rightarrow \pi^{-1} \pi_{!} F \rightarrow \pi^{-1} G \subset \pi^{-1} \pi_{!}^{\mathbf{N}} F
$$

Of course, the first map is just a morphism of sheaves of sets, but the composition is a monoid homomorphism, so upon applying $\pi_{!}^{\mathbf{N}}$ again, we get a commutative diagram

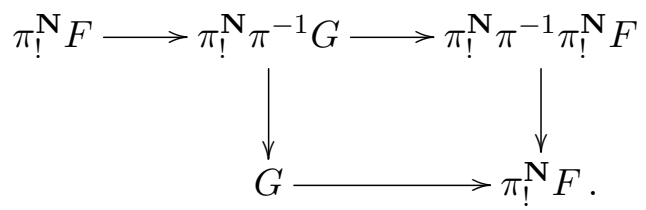

Adjunction implies that the composition $\pi_{!}^{\mathbf{N}} F \rightarrow \pi_{!}^{\mathbf{N}} F$ is the identity, from which it follows that $G \rightarrow \pi_{!}^{\mathbf{N}} F$ is surjective. This proves the first claim.

For the second, consider a surjection $H \rightarrow F$ of sheaves of integral monoids. Then $\pi_{!} H \rightarrow \pi_{!} F$ is surjective, and $\pi_{!} F$ generates $\pi_{!}^{\mathbf{N}} F$, so the image of $\pi_{!} H$ in $\pi_{!}^{\mathbf{N}} F$ generates $\pi_{!}^{\mathbf{N}} F$. Therefore, $\pi_{!}^{\mathbf{N}} H \rightarrow \pi_{!}^{\mathbf{N}} F$ is surjective. 


\section{UNIQUENESS OF MINIMAL MORPHISMS}

Lemma 4.3. Let $i: \underline{U} \rightarrow \underline{X}$ be the inclusion of an open subset such that $\underline{U}_{s} \subset \underline{X}_{s}$ is dense for every geometric point $s$ of $\underline{S}$.

(i) For any étale sheaf of sets $F$ on $\underline{X}$, the map $\pi_{!} i_{!} i^{-1} F \rightarrow \pi_{!} F$ is surjective.

(ii) For any étale sheaf of integral monoids $F$ on $\underline{X}$, the homomorphism $\pi_{!}^{\mathbf{N}} i_{!}^{\mathbf{N}} i^{-1} F \rightarrow \pi_{!}^{\mathbf{N}} F$ is surjective.

Proof. We begin with the statement about sheaves of sets. Since $\pi_{\text {! }}$ commutes with base change and surjectivity can be checked on the stalks, it is sufficient to assume that $\underline{S}$ is the spectrum of an algebraically closed field. By Lemma 4.1, we have

$$
\begin{aligned}
\pi_{!} F & =\pi_{0}\left(F^{\text {ét }}\right), \\
\pi_{!} i_{!} i^{-1} F & =\pi_{0}\left(i^{-1} F^{\text {ét }}\right) .
\end{aligned}
$$

But $\underline{X}$ is locally connected (since it is locally of finite type over a field) and $i^{-1} F^{\text {ét }} \subset F^{\text {ét }}$ is a dense open subset, so $\pi_{0}\left(i^{-1} F^{\text {ét }}\right)$ surjects onto $\pi_{0}\left(F^{\text {ét }}\right)$.

Now, we prove the statement about monoids. As before, let $\mathbf{N} F$ be the sheaf of monoids freely generated by the underlying sheaf of sets of $F$; note that $\mathbf{N} F \rightarrow F$ is surjective. Consider the commutative diagram

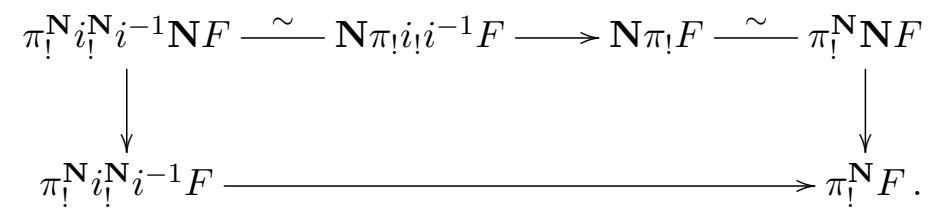

The upper arrow is surjective by the first part of the lemma, and Lemma 4.2 implies that the right arrow is surjective, so the bottom arrow is surjective as well.

\section{Construction of minimal monoids}

We recall the notation and main construction of [Wis16]. A simple example of this construction can be found in Section 8.

Recall our assumptions from Section 2, to which we add that

(iii) $\pi$ is flat with reduced geometric fibers and

(iv) $\pi^{*} M_{S} \rightarrow M_{X}$ is integral.

We also specify a type, $u$.

We think of fibered categories over LogSch as fibered categories over Sch via the projection $\mathbf{L o g S c h} \rightarrow \mathbf{S c h}$. Thus, if $H$ is a fibered category and $\underline{S}$ is a scheme, the notation $H(\underline{S})$ refers to the category of all pairs $\left(M_{S}, \xi\right)$ where $M_{S} \in \operatorname{LogSch}(\underline{S})$ is a logarithmic structure on $S$ and $\xi \in H\left(\underline{S}, M_{S}\right)$.

In order to describe more concretely the particular choice of $H$ that will be of interest to us, we introduce some notation. Let $f: T \rightarrow S$ be a morphism of logarithmic schemes, and set $\underline{Y}=\underline{X} \times \underline{S} \underline{T}$. For any logarithmic structure $P$ on $X$, we write $f^{*} P$ for the logarithmic structure on $\underline{Y}$ such that $\left(\underline{Y}, f^{*} P\right)=(\underline{X}, P) \times_{S} T$ in the category of (not necessarily fine) logarithmic schemes; that is, $f^{*} P$ is obtained by pushing out $f^{-1} P$ along the morphism $\pi^{-1} f^{-1} M_{S} \rightarrow \pi^{-1} M_{T}$ and taking the associated logarithmic structure. 


\section{J. WISE}

Define $H=\operatorname{Hom}_{\mathbf{L o g S c h} / S}\left(M, M_{X}\right)_{u}$. This is the fibered category over $\mathbf{L o g S c h} / S$ whose fiber over $f:\left(T, M_{T}\right) \rightarrow\left(S, M_{S}\right)$ is the set of morphisms of logarithmic structures

$$
f^{*} M \rightarrow f^{*} M_{X}
$$

on $\underline{X} \times \underline{S} \underline{T}$ that commute with the structural maps from $f^{*} \pi^{*} M_{S}=\pi^{*} M_{T}$ and such that the induced map

$$
f^{*} M^{\mathrm{gp}} / f^{*} \pi^{*} M_{S}^{\mathrm{gp}} \rightarrow f^{*} M_{X}^{\mathrm{gp}} / f^{*} \pi^{*} M_{S}^{\mathrm{gp}}
$$

coincides with the pullback $f^{*} u$ of the type $u$. Note that $\underline{X} \times \underline{S} \underline{T}$ is the underlying scheme of $X \times{ }_{S} T$ because $X$ is integral over $S$.

We will often wish to refer to objects of the moduli space $H$ with some additional fixed structure. For example, to fix a logarithmic base scheme $T$, we write $H(T)$; to fix the underlying scheme $\underline{T}$ of $T$ and its characteristic monoid $\bar{N}_{T}$ (but not the logarithmic structure $N_{T}$ ), we write $H\left(\underline{T}, \bar{N}_{T}\right)$.

It was shown in [Wis16, Theorem 1.1] that if $\underline{X}$ is proper over $\underline{S}$, then $H$ is representable by an algebraic space $\underline{H}$ with a logarithmic structure $M_{H}$, that is locally of finite presentation over $\underline{S}$. By a theorem of Gillam, $\underline{H}(\underline{S}) \subset H(\underline{S})$ may be characterized as the subcategory of minimal objects (see [Gil12] or [Wis16, Appendix B]). This category is equivalent to a set by [Wis16, Corollary 5.1.1], which shows that minimal objects have no nontrivial automorphisms.

We will be particularly interested in the $\underline{S}$-points of $H$, so we introduce some additional notation for handling them. For any morphism of logarithmic structures $M_{S} \rightarrow N_{S}$, we write $M_{X} \rightarrow N_{X}$ for morphism of logarithmic structures on $X$ obtained by pushout along the morphism $\pi^{*} M_{S} \rightarrow M_{X}$ :

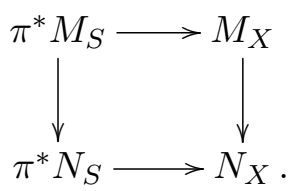

In other words, $\left(\underline{X}, N_{X}\right)=X \times{ }_{S}\left(\underline{S}, N_{S}\right)$.

Then $H(\underline{S})$ is the category of pairs $\left(M_{S} \rightarrow N_{S}, M \stackrel{\varphi}{\rightarrow} N_{X}\right)$, where $N_{X}$ is as above and and $M \rightarrow N_{X}$ is a morphism of logarithmic structures commuting with the maps from $\pi^{*} M_{S}$. Such an object is generally abbreviated to $\left(N_{S}, \varphi\right)$.

We now recall the construction of the logarithmic structure of $H$ from [Wis16]. This can be done without explicit reference to the underlying space of $H$ : given an $\underline{S}$-point $\left(N_{S}, \varphi\right)$ of $H$, there is a corresponding map $\alpha: \underline{S} \rightarrow \underline{H}$; there must therefore be a logarithmic structure $\alpha^{*} M_{H}$ on $\underline{S}$ and a homomorphism of logarithmic structures $\alpha^{*} M_{H} \rightarrow N_{S}$; we build the logarithmic structure $\alpha^{*} M_{H}$. This is known as the minimal (or basic) logarithmic structure associated with $\left(N_{S}, \varphi\right)$.

In fact, what we do is find a sheaf of monoids (a quasilogarithmic structure, in the language of [Wis16, Definition 1.2]) $\bar{Q}_{S}$ on $\underline{S}$ such that $\alpha^{-1} \bar{M}_{H}$ is a quotient of $\bar{Q}_{S}$. We recall the construction of $\bar{Q}_{S}$.

First, we form a fiber product:

$$
\bar{R}_{0}^{\mathrm{gp}}=\bar{M}^{\mathrm{gp}} \underset{M_{X / S}^{\mathrm{gp}}}{\times} \bar{M}_{X}^{\mathrm{gp}} .
$$

The first map in the product is the type $u$, and the second is the tautological projection. The fiber product comes with two inclusions of $\pi^{-1} \bar{M}_{S}^{\mathrm{gp}}$, one from each factor, and we take $\bar{R}^{\text {gp }}$ to 


\section{UNIQUENESS OF MINIMAL MORPHISMS}

be their coequalizer. Then, we define the submonoid $\bar{R} \subset \bar{R}^{\text {gp }}$ to be the smallest submonoid containing $\pi^{-1} \bar{M}_{S}$ such that the pushout $\bar{R}_{X}$ in diagram (5.3)

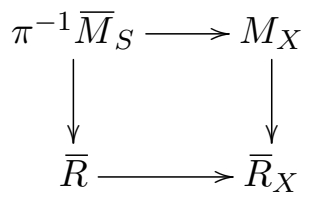

contains the image of $\bar{M}$ under the tautological map (see [Wis16, Section 3.1] for the construction of this map). Next, we consider the pushout

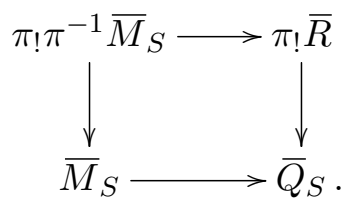

It was shown in [Wis16, Section 4.2] that if $\left(N_{S}, \varphi\right) \in H(S)$, then there is a unique homomorphism of sheaves of monoids, commuting with maps from $\bar{M}_{S}$ and inducing $\bar{\varphi}: \bar{M} \rightarrow \bar{N}_{X}$ :

$$
\bar{Q}_{S} \rightarrow \bar{N}_{S} \text {. }
$$

Pulling back the projection $N_{S} \rightarrow \bar{N}_{S}$ and the map $N_{S} \rightarrow \mathcal{O}_{S}$ induces a sheaf of monoids $Q_{S}$ on $\underline{S}$ and a homomorphism $Q_{S} \rightarrow \mathcal{O}_{S}$. This is not always a logarithmic structure on $S$ (see, for example, the case of logarithmic points in Section 8), so we pass to the associated logarithmic structure to get $\alpha^{*} M_{H}$.

We record several basic consequences of the construction.

LEMMA 5.1. (i) $\bar{Q}_{S}^{\mathrm{gp}} / \bar{M}_{S}^{\mathrm{gp}} \simeq \pi_{!} \bar{R}^{\mathrm{gp}} / \pi_{!} \pi^{-1} \bar{M}_{S}^{\mathrm{gp}} \simeq \pi_{!}\left(\bar{R}^{\mathrm{gp}} / \pi^{-1} \bar{M}_{S}^{\mathrm{gp}}\right)$.

(ii) $\bar{R}^{\mathrm{gp}} / \pi^{-1} \bar{M}_{S}^{\mathrm{gp}} \simeq \bar{R}_{0}^{\mathrm{gp}} /\left(\pi^{-1} \bar{M}_{S}^{\mathrm{gp}} \times \pi^{-1} \bar{M}_{S}^{\mathrm{gp}}\right) \simeq \bar{M}^{\mathrm{gp}} / \pi^{-1} \bar{M}_{S}^{\mathrm{gp}}$.

(iii) The sharpening of $\bar{Q}_{S} \rightarrow \bar{N}_{S}$ is $\alpha^{-1} \bar{M}_{H}$.

(iv) If $M_{X / S}=0$, then $\bar{R}=\bar{M}$, canonically.

Proof. Passage to the associated group is a left adjoint and therefore preserves cocartesian diagrams. Therefore, diagram (5.4) remains cocartesian upon passage to the associated groups. It is then an easy exercise with universal properties to verify that the quotients along the horizontal direction are isomorphic. This gives the isomorphism on the left side of statement (i); for the right side, we observe that $\pi_{\text {! }}$ is a left adjoint, hence respects quotients.

For statement (ii), observe that to go from the middle term to the left one, we quotient both $\bar{R}_{0}^{\mathrm{gp}}$ and $\pi^{-1} \bar{M}_{S}^{\mathrm{gp}} \times \pi^{-1} \bar{M}_{S}^{\mathrm{gp}}$ by the antidiagonal copy of $\pi^{-1} \bar{M}_{S}^{\mathrm{gp}}$. To go from middle to right, we use the fiber product construction of $\bar{R}_{0}^{\mathrm{gp}}$ in (5.2) and quotient by the right copy of $\pi^{-1} \bar{M}_{S}^{\mathrm{gp}}$.

For statement (iii), first recall that the sharpening of $\bar{Q}_{S} \rightarrow \bar{N}_{S}$ is the minimal quotient $\bar{Q}_{S}^{\prime}$ of $\bar{Q}_{S}$ through which the map to $\bar{N}_{S}$ factors as a sharp homomorphism. It is constructed by dividing $\bar{Q}_{S}$ by the set of elements that map to 0 in $\bar{N}_{S}$.

Now, $Q_{S}$ is, by construction, an extension of $\bar{Q}_{S}$ by $\mathcal{O}_{S}^{*}$. By definition, the associated logarithmic structure $Q_{S}^{a}$ of $Q_{S}$ fits into a cocartesian diagram:

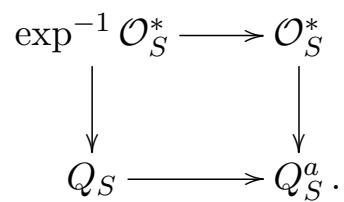




\section{J. WISE}

But note that $\exp ^{-1} \mathcal{O}_{S}^{*}=\gamma^{-1} N_{S}^{*}$, where $\gamma: Q_{S} \rightarrow N_{S}$ is the tautological map. Dividing everything by $\mathcal{O}_{S}^{*}$ yields another cocartesian diagram:

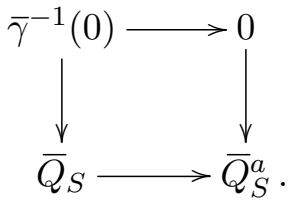

On the other hand, this is precisely the cocartesian diagram used to sharpen $\bar{Q}_{S} \rightarrow \bar{N}_{S}$.

For statement (iv), observe that when $M_{X / S}=0$, the fiber product (5.2) becomes a product: $\bar{R}_{0}^{\mathrm{gp}}=\bar{M}^{\mathrm{gp}} \times \pi^{-1} \bar{M}_{S}^{\mathrm{gp}}$, so equalizing the two copies of $\pi^{-1} \bar{M}_{S}^{\mathrm{gp}}$ recovers $\bar{M}^{\mathrm{gp}}$. Chasing the definitions $^{3}$ in [Wis16, Section 3.1], one discovers that the map $\bar{M}^{\mathrm{gp}} \rightarrow \bar{R}_{X}=\bar{M}^{\mathrm{gp}}$ is the identity under this identification, and therefore that $\bar{R}=\bar{M}$.

\section{Minimal characteristic monoids}

We maintain the assumptions of Section 2 and the additional assumptions of Section 5. Recall that by our notational conventions, $H(\underline{S})$ consists of all choices of logarithmic structure $N_{S}$ on $\underline{S}$ and all $\varphi \in H\left(\underline{S}, N_{S}\right)$.

Lemma 6.1. Let $\bar{R}$ be constructed as in Section 5. If the relative characteristic of $X / S$ is generically trivial on every geometric fiber of $X$ over $S$, then for any $\left(N_{S}, \varphi\right) \in H(\underline{S})$, the canonical map $\pi_{!} \bar{R} \rightarrow \bar{N}_{S}$ is sharp.

Proof. Since the construction of $\pi_{!} \bar{R}$ commutes with change of base, we can reduce to the case where $\bar{S}$ is the spectrum of an algebraically closed field. By Lemma 4.3, there is a surjection $\pi_{!} i_{!} i^{-1} \bar{R} \rightarrow \pi_{!} \bar{R}$, where $i$ is the inclusion of the dense open subset where $M_{X / S}=0$. It is sufficient to show that $\pi_{!} i_{!} i^{-1} \bar{R} \rightarrow \bar{N}_{S}$ is sharp. We can therefore reduce to the case where $M_{X / S}=0$, globally, by replacing $X$ with the dense open subset on which the characteristic monoid vanishes.

In that case, $\bar{R}=\bar{M}$ (Lemma 5.1(iv)) and the map $\pi_{!} \bar{R} \rightarrow \bar{N}_{S}$ is induced by adjunction from the map

$$
\bar{\varphi}: \bar{M} \rightarrow \bar{N}_{X}=\pi^{-1} \bar{N}_{S} .
$$

The equality on the right holds because $M_{X / S}=0$. But now by Lemmas 4.1 and $4.2(\mathrm{i})$, any $a \in \pi_{!} \bar{M}$ is the image of a sum of local sections $a_{i}$ of $\bar{M}$ defined over connected $\underline{U}_{i}$ that are étale over $\underline{X}$. If $a=\sum a_{i}$ maps to 0 in $\bar{N}_{S}$, then all $a_{i}$ map to 0 in $\bar{N}_{S}$ since $\bar{N}_{S}$ is sharp (as it is the characteristic monoid of a logarithmic structure). This means that $a_{i}$ maps to 0 under $\bar{\varphi}: \bar{M} \rightarrow \pi^{-1} \bar{N}_{S}$. But $\bar{\varphi}$ underlies the morphism of logarithmic structures $\varphi: M \rightarrow \pi^{*} N_{S}$, hence is sharp. Therefore, all of the $a_{i}$ must be 0 , so $\pi_{!} \bar{R} \rightarrow \bar{N}_{S}$ is sharp.

Corollary 6.2. Under the hypotheses of the lemma, if $\left(N_{S}, \varphi\right) \in \underline{H}(\underline{S})$, then $\bar{N}_{S}=\bar{Q}_{S}$.

Proof. By Lemma 5.1(iii), the monoid $\bar{N}_{S}$ is the sharpening of $\bar{Q}_{S} \rightarrow \bar{N}_{S}$, so the point is to show that $\bar{Q}_{S} \rightarrow \bar{N}_{S}$ is sharp. We already know that $\pi_{!} \bar{R} \rightarrow \bar{N}_{S}$ is sharp by the lemma. When $\underline{X}$ has connected geometric fibers over $\underline{S}$, this implies the corollary since in that case $\pi_{!} \bar{R}=\bar{Q}_{S}$.

\footnotetext{
${ }^{3}$ The reader who is so inclined may prefer to observe that $\bar{R}$ and $\bar{M} \rightarrow \bar{R}_{X}$ (where $\bar{R}_{X}$ is the pushout of $\pi^{-1} \bar{M}_{S} \rightarrow$ $\bar{M}_{X}$ along $\pi^{-1} \bar{M}_{S} \rightarrow \bar{R}$ ) satisfy a universal property and that $\bar{M}$ and id: $\bar{M} \rightarrow \bar{M}$ visibly satisfy this universal property when $M_{X / S}=0$.
} 


\section{UNIQUENESS OF MINIMAL MORPHISMS}

In general, we can proceed geometric fiber by geometric fiber and assume that $\underline{S}$ is the spectrum of an algebraically closed field. If $\underline{X}=\varnothing$, the conclusion is obvious. Otherwise, the map $\pi_{!} \pi^{-1} \bar{M}_{S} \rightarrow \bar{M}_{S}$ is surjective, which implies that $\pi_{!} \bar{R} \rightarrow \bar{Q}_{S}$ is surjective as well. Therefore, the sharpness of $\pi_{!} \bar{R} \rightarrow \bar{N}_{S}$ implies the sharpness of $\bar{Q}_{S} \rightarrow \bar{N}_{S}$, as required.

The corollary implies that all objects of $\underline{H}(\underline{S})$ of the same type $u$ have the same characteristic monoid. This greatly simplifies the analysis of $\underline{H}_{u}(\underline{S})$. In fact, the following lemma gives a complete characterization of $H_{u}\left(\underline{S}, \bar{N}_{S}\right)$ for any fixed characteristic monoid $\bar{N}_{S}$.

Lemma 6.3. Assume that $\underline{S}$ is the spectrum of an algebraically closed field. If $H_{u}\left(\underline{S}, \bar{N}_{S}\right)$ is nonempty, then it is isomorphic to the quotient groupoid

$$
\left[\operatorname{Hom}\left(\bar{M}^{\mathrm{gp}} / \pi^{-1} \bar{M}_{S}^{\mathrm{gp}}, \mathcal{O}_{X}^{*}\right) / \operatorname{Hom}\left(\bar{N}_{S}^{\mathrm{gp}} / \bar{M}_{S}^{\mathrm{gp}}, \mathcal{O}_{S}^{*}\right)\right] .
$$

The map $\operatorname{Hom}\left(\bar{N}_{S} / \bar{M}_{S}, \mathcal{O}_{S}^{*}\right) \rightarrow \operatorname{Hom}\left(\bar{M} / \pi^{-1} \bar{M}_{S}, \mathcal{O}_{X}^{*}\right)$ used to construct the quotient is obtained from the canonical maps

$$
\begin{aligned}
\pi^{-1} \mathcal{O}_{S}^{*} & \rightarrow \mathcal{O}_{X}^{*}, \\
\bar{M}^{\mathrm{gp}} / \pi^{-1} \bar{M}_{S}^{\mathrm{gp}} & \rightarrow \bar{N}_{X}^{\mathrm{gp}} / \bar{M}_{X}^{\mathrm{gp}} \simeq \pi^{-1} \bar{N}_{S}^{\mathrm{gp}} / \pi^{-1} \bar{M}_{S}^{\mathrm{gp}},
\end{aligned}
$$

the latter of which is induced from $\bar{\varphi}$ and the cocartesian diagram (5.1).

Proof. We need to see how many ways there are to choose $\left(N_{S}, \varphi\right) \in H_{u}(\underline{S})$ with the same fixed characteristic monoid $\bar{N}_{S}$. Holding $N_{S}$ fixed, any two choices of $\varphi$ will differ by a uniquely determined homomorphism $\bar{M} \rightarrow \mathcal{O}_{X}^{*}$ that vanishes on $\pi^{-1} \bar{M}_{S}$. Therefore, for $N_{S}$ fixed, the choices of $\varphi$ form a torsor under $\operatorname{Hom}\left(\bar{M} / \pi^{-1} \bar{M}_{S}, \mathcal{O}_{X}^{*}\right)$.

Since $S$ is the spectrum of an algebraically closed field, there is a unique choice of $N_{S}$ (up to nonunique isomorphism) once $\bar{N}_{S}$ is fixed. Making such a choice, we can now identify $H_{u}\left(\underline{S}, \bar{N}_{S}\right)$ with the quotient of $\operatorname{Hom}\left(\bar{M} / \pi^{-1} \bar{M}_{S}, \mathcal{O}_{X}^{*}\right)$ by the automorphism group of $N_{S}$ fixing $\bar{N}_{S}$ and $M_{S}$. That automorphism group is precisely $\operatorname{Hom}\left(\bar{N}_{S} / \bar{M}_{S}, \mathcal{O}_{S}^{*}\right)$, by the same calculation we made above.

Putting these two lemmas together, we discover first from Lemma 6.1 that if $H_{u}(\underline{S}) \neq \varnothing$, then the characteristic monoid of any object of $\underline{H}_{u}(\underline{S})$ is $Q_{S}$. Then Lemma 6.3 implies that $\underline{H}_{u}(\underline{S})$ may be identified with the quotient of $\operatorname{Hom}\left(\bar{M}^{\mathrm{gp}} / \pi^{-1} \bar{M}_{S}^{\mathrm{gp}}, \mathcal{O}_{X}^{*}\right)$ by

$$
\begin{aligned}
\operatorname{Hom}\left(\bar{Q}_{S}^{\mathrm{gp}} / \bar{M}_{S}^{\mathrm{gp}}, \mathcal{O}_{S}^{*}\right) & =\operatorname{Hom}\left(\pi_{!} \bar{R}^{\mathrm{gp}} / \pi_{!} \pi^{-1} \bar{M}_{S}^{\mathrm{gp}}, \mathcal{O}_{S}^{*}\right) \\
& =\operatorname{Hom}\left(\bar{R}^{\mathrm{gp}} / \pi^{-1} \bar{M}_{S}^{\mathrm{gp}}, \pi^{-1} \mathcal{O}_{S}^{*}\right) \\
& =\operatorname{Hom}\left(\bar{M}^{\mathrm{gp}} / \pi^{-1} \bar{M}_{S}^{\mathrm{gp}}, \pi^{-1} \mathcal{O}_{S}^{*}\right) \quad(\text { Lemma } 5.1(\mathrm{ii})) .
\end{aligned}
$$

We will therefore be able to conclude that the quotient $\underline{H}(\underline{S})$ is trivial once we prove

$$
\operatorname{Hom}\left(\bar{M}^{\mathrm{gp}} / \pi^{-1} \bar{M}_{S}^{\mathrm{gp}}, \mathcal{O}_{X}^{*}\right)=\operatorname{Hom}\left(\bar{M}^{\mathrm{gp}} / \pi^{-1} \bar{M}_{S}^{\mathrm{gp}}, \pi^{*} \mathcal{O}_{S}^{*}\right) \text {. }
$$

In other words, we must show that every homomorphism

$$
\bar{M}_{X}^{\mathrm{gp}} / \pi^{-1} \bar{M}_{S}^{\mathrm{gp}} \rightarrow \mathcal{O}_{X}^{*}
$$

factors through $\pi^{-1} \mathcal{O}_{S}^{*}$. That will be done in the next section. 


\section{J. WISE}

\section{Units}

Theorem 7.1. Suppose that $X$ is reduced, that $X$ is proper over $S$, and that $M$ is a coherent logarithmic structure on $X$. Then any homomorphism $\bar{M} \rightarrow \mathcal{O}_{X}^{*}$ factors through $\pi^{-1} \mathcal{O}_{S}^{*}$.

Remark. Theorem 7.1 is obvious in the special case where $\bar{M}^{\mathrm{gp}}$ is generated by global sections, since maps $\bar{M} \rightarrow \mathcal{O}_{X}^{*}$ correspond to global sections of $\mathcal{O}_{X}^{*}$.

For any scheme $X$, let $\mathscr{U} \subset \mathcal{O}_{X}^{*}$ be the étale subpresheaf ${ }^{4}$ whose sections over an étale $V \rightarrow X$ consist of all $f \in \mathcal{O}_{X}^{*}(V)$ such that, for every valuation ring $R$ with field of fractions $K$ and every commutative diagram

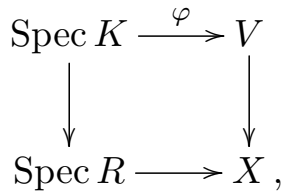

the restriction $\varphi^{*} f$ of $f$ to $\operatorname{Spec} K$ lies in $R^{*}$. The idea is that $\mathscr{U}(V)$ is the sheaf of units in $\mathscr{O}_{X}^{*}(V)$ that have no zeroes or poles on the closure of $V$.

Lemma 7.2. The presheaf $\mathscr{U}$ defined above is a sheaf in the étale topology.

Proof. As $\mathscr{U}$ is a subpresheaf of a sheaf, it is separated. We need to show that every local section of $\mathscr{O}_{X}^{*}$ that lies locally in $\mathscr{U}$ is in fact in $\mathscr{U}$. Suppose that we have an étale cover $W_{i} \rightarrow V$ and that $f$ is a section of $\mathcal{O}_{X}^{*}(V)$ whose restriction to each $W_{i}$ lies in $\mathscr{U}\left(W_{i}\right)$. We check that $f$ lies in $\mathscr{U}(V)$.

To that end, suppose that $R$ is a valuation ring with field of fractions $K$ and that we have a commutative diagram (7.1). Then, there is some $W_{i}$ whose image in $V$ contains the image of Spec $K$. Let $K_{1}$ be the residue field of a finite type point of $V_{i} \times_{V}$ Spec $K$. Since $K_{1}$ is finite over $K$, there is a unique extension of the valuation of $K$ to $K_{1}$. Let $R_{1}$ be its valuation ring. We have a commutative diagram

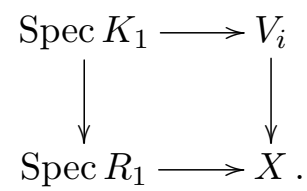

As $f \in \mathscr{U}\left(V_{i}\right)$, we deduce that the image of $f$ in $K_{1}$ lies in $R_{1}^{*}$. But $R_{1}^{*} \cap K=R^{*}$, so $f \in \mathscr{U}(V)$ as well.

Lemma 7.3. Suppose that $M$ is a coherent logarithmic structure on $X$. Then any homomorphism of sheaves of monoids $\bar{M} \rightarrow \mathcal{O}_{X}^{*}$ factors through $\mathscr{U}$.

Proof. Let $\alpha: \bar{M} \rightarrow \mathcal{O}_{X}^{*}$ be a homomorphism. Consider a section $f \in \bar{M}(V)$ for some étale $V \rightarrow X$. We must show that for any commutative diagram (7.1), the image of $\varphi^{*} \alpha$ in $K^{*}$ lies in $R^{*}$. We can therefore replace $X$ with $\operatorname{Spec} R$ and $V$ with $\operatorname{Spec} K$. Since $K^{*}$ and $R^{*}$ are sheaves in the étale topology on $\operatorname{Spec} R$ (to be more precise, $\mathcal{O}_{\operatorname{Spec} R}^{*}$ and $j_{*} \mathcal{O}_{\text {Spec } K}^{*}$ are sheaves, where $j$ is the inclusion of the generic point), we can work étale-locally in $X$ and assume that $M$ has a global chart. Then, the image of $\alpha$ coincides with the image of the global sections of $\bar{M}$ under $\alpha$, and any global section of $\bar{M}$ maps to $\Gamma\left(X, \mathcal{O}_{X}^{*}\right)=R^{*}$.

${ }^{4}$ I do not know whether it is necessary to work in the étale topology, or if the sheaf is induced from the Zariski topology. 


\section{UNIQUENESS OF MINIMAL MORPHISMS}

Lemma 7.4. When $\pi: X \rightarrow S$ is proper and $X$ is reduced, the natural inclusion $\pi^{-1} \mathcal{O}_{S}^{*} \subset \mathscr{U}$ is a bijection.

Proof. Suppose that $f$ is a section of $\mathscr{U}$ over an étale map $V \rightarrow X$. We can assume that $V$ is quasicompact. Regard $f$ as a morphism from $X$ to $\mathbf{G}_{m} \subset \mathbf{P}^{1}$, let $\bar{V}$ be the closure of the graph of $f$ in $X \times \mathbf{P}^{1}$ (with its reduced structure), and let $\bar{f}: \bar{V} \rightarrow \mathbf{P}^{1}$ be the projection. For any point $q$ of $\bar{V}$, we can choose a valuation of $\mathcal{O}_{\bar{V}}$ whose center is $q$ and whose generic point lies in $V$; let $R$ be the valuation ring. Then, by the definition of $\mathscr{U}$, the restriction of $\bar{f}$ to Spec $R$ lies in $R^{*}$. In particular, $\bar{f}(q) \neq 0, \infty$. Therefore, $\bar{f}$ factors through $\mathbf{G}_{m} \subset \mathbf{P}^{1}$. This holds for any $q \in \bar{V}$, so $\bar{f} \in \Gamma\left(\bar{V}, \mathcal{O}_{\bar{V}}^{*}\right)$. But $\bar{V}$ is reduced and proper over $S$, so $\Gamma\left(\bar{V}, \mathcal{O}_{\bar{V}}^{*}\right)=\Gamma\left(\bar{V}, \pi^{-1} \mathcal{O}_{S}^{*}\right)$. And as $V$ is reduced (since it is étale over $X$ and $X$ is reduced), we have $V \subset \bar{V}$ as a scheme and $f$ is the restriction of $\bar{f}$ to $V$. Thus $f \in \Gamma\left(V, \pi^{-1} \mathcal{O}_{S}^{*}\right)$, as required.

Proof of Theorem 7.1. By Lemma 7.3, any homomorphism $\bar{M} \rightarrow \mathcal{O}_{X}^{*}$ factors through $\mathscr{U}$. But because $X$ is reduced and proper over $S$, Lemma 7.4 implies $\mathscr{U}=\pi^{-1} \mathcal{O}_{S}^{*}$.

Proof of Theorem 2.2. By Corollary 6.2, any $\left(N_{S}, \varphi\right) \in \underline{H}_{u}(\underline{S})$ must have $\underline{N}_{S}=Q_{S}$. Then by Lemma 6.3 , if there are any objects of $H(\underline{S})$ with characteristic monoid $\underline{Q}_{S}$, then they are parameterized by the quotient

$$
\left[\operatorname{Hom}\left(\underline{M}^{\mathrm{gp}} / \pi^{-1} \bar{M}_{S}^{\mathrm{gp}}, \mathcal{O}_{X}^{*}\right) / \operatorname{Hom}\left(\underline{Q}_{S}^{\mathrm{gp}} / \bar{M}_{S}^{\mathrm{gp}}, \mathcal{O}_{S}^{*}\right)\right] .
$$

The chain of equalities at the end of Section 6 and Theorem 7.1 imply that

$$
\operatorname{Hom}\left(\bar{Q}_{S}^{\mathrm{gp}} / \bar{M}_{S}^{\mathrm{gp}}, \mathcal{O}_{S}^{*}\right)=\operatorname{Hom}\left(\bar{M}^{\mathrm{gp}} / \pi^{-1} \bar{M}_{S}^{\mathrm{gp}}, \mathcal{O}_{X}^{*}\right),
$$

so the quotient (7.2) is trivial.

\section{A counterexample: Logarithmic points}

It is perhaps easiest to appreciate how the criterion of Theorem 1.1 works by studying why quasifiniteness fails in an example where the criterion does not apply.

We consider the moduli space of logarithmic points valued in the standard logarithmic point, considered by Abramovich, Chen, Gillam, and Marcus [Gil12, ACGM10]: Let $k$ be the spectrum of an algebraically closed field, let $\underline{S}=$ Spec $k$ and $M_{S}=0$, and let $X$ and $Y$ both be the standard logarithmic point over $k$, both regarded as logarithmic schemes over $S$. The space we are interested in is $\operatorname{Hom}_{\operatorname{LogSch} / S}(X, Y)$. Since the underlying map of schemes $\underline{X} \rightarrow \underline{Y}$ must be the identity, this may be identified with $\operatorname{Hom}_{\mathbf{L o g S c h} / S}\left(M, M_{X}\right)$, where $M=M_{Y}=M_{X}=\mathbf{N} \times k^{*}$.

An $S$-point of this moduli space is simply a map of logarithmic structures $M \rightarrow M_{X}$, and our choices are determined by where the generator $(1,1)$ goes. That gives $\mathbf{N} \times k^{*}$ for the $S$-points.

We could also look at the construction of the minimal monoid associated with one of these maps. Following the algorithm from Section 5, we should form $\bar{R}^{\mathrm{gp}}=\bar{M}^{\mathrm{gp}} \times_{M_{X / S}} \bar{M}_{X}^{\mathrm{gp}}$. Since $\bar{M}_{S}$ is trivial, this is just $\bar{M}^{\mathrm{gp}}$. (There is an additional quotient by $\bar{M}_{S}^{\mathrm{gp}}$ in the algorithm which does not change anything since $\bar{M}_{S}=0$.) Then we identify the smallest submonoid $\bar{R} \subset \bar{R}^{\text {gp }}$ such that $\bar{R}_{X}=\bar{M} \times \mathbf{N}$ contains the image of $\bar{M}$ under the tautological map (id, $\left.u\right): \bar{M} \rightarrow \bar{M}^{\mathrm{gp}} \times \mathbf{Z}$ (here $u$ is the type). This submonoid is obviously $\bar{M}$ itself, so $\bar{M}$ is the minimal characteristic monoid.

In order to obtain an actual logarithmic structure, we need to assume that $u$ came from an actual logarithmic map over some $\left(\underline{S}, N_{S}\right)$. This induces a map $\bar{M} \rightarrow \bar{N}_{S}$ since $\bar{M}$ is minimal 


\section{J. WISE}

on the level of characteristic monoids (see Section 5). This gives a quasilogarithmic structure (see [Wis16, Definition 1.2]) by pulling back $N_{S}$ to $\bar{M}$, but in order to get a genuine logarithmic structure, we must also sharpen the map $\bar{M} \rightarrow \bar{N}_{S}$; that is, we must divide $\bar{M}$ by the preimage of 0 .

In our example, we are working over $\left(S, \mathcal{O}_{S}^{*}\right)$, so that $\bar{N}_{S}=0$. Therefore, the sharpening of the minimal quasilogarithmic structure is trivial.

The main observation of this paper was thus that the sharpening process is responsible for the failure of quasifiniteness. More precisely, when the sharpening process does not change anything, there is at most one choice (up to unique isomorphism) of a minimal object (at least if the domain is proper). Indeed, Corollary 6.2 and Lemma 6.3 show that (under an assumption of properness) the automorphism group of the minimal quasilogarithmic structure $Q_{S}$ as an extension of its characteristic monoid by $\mathcal{O}_{S}^{*}$ - but not respecting the map to $\mathcal{O}_{S}$ - precisely cancels the choices of maps $M \rightarrow Q_{X}$. This automorphism group agrees with the automorphism group of the minimal logarithmic structure exactly when $Q_{S}$ is the minimal logarithmic structure, and this occurs when the map $\pi_{!} \bar{R} \rightarrow \bar{N}_{S}$ is sharp (Lemma 5.1(iii)).

Thus, the analysis of the fiber of the moduli space of logarithmic maps comes down to the question of whether the minimal quasilogarithmic structure $Q_{S}$ is already a logarithmic structure. Lemma 6.1 shows that a sufficient condition is that the relative characteristic monoid $M_{X / S}$ be generically trivial.

\section{ACKNOWLEDGEMENTS}

This paper was born out of a joint project with D. Abramovich, Q. Chen, and S. Marcus to prove the same result for stable maps of logarithmic curves using a different method. While my collaborators aver they have not contributed to the present paper and prefer not to be included as coauthors, I could not have written this paper without the intuition gained from the many examples we studied together. I am very grateful to them for this, and for their generosity in allowing me to take credit for the solution. I am particularly grateful to Dan Abramovich, whose comments improved the exposition of this paper considerably.

I would also like to thank M. Chan for a suggestion that was very useful in our original approach to this problem, even though the method presented here circumvents it. I am grateful as well to the referees for the care they have taken with the paper, and for their corrections.

\section{REFERENCES}

AC14 D. Abramovich and Q. Chen, Stable logarithmic maps to Deligne-Faltings pairs II, Asian J. Math. 18 (2014), no. 3, 465-488; doi:10.4310/AJM.2014.v18.n3.a5.

ACGM10 D. Abramovich, Q. Chen, W. D. Gillam, and S. Marcus, The evaluation space of logarithmic stable maps, 2010, arXiv:1012.5416.

ACMW17 D. Abramovich, Q. Chen, S. Marcus, and J. Wise, Boundedness of the space of stable logarithmic maps, J. Eur. Math. Soc. 19 (2017), no. 9, 2783-2809; doi:10.4171/JEMS/728.

Che14 Q. Chen, Stable logarithmic maps to Deligne-Faltings pairs I, Ann. of Math. 180 (2014), no. 2, 455-521; doi:10.4007/annals.2014.180.2.2.

Gil12 W. D. Gillam, Logarithmic stacks and minimality, Internat. J. Math. 23 (2012), no. 7, 1250069, 38 pp.; doi:10.1142/S0129167X12500693. 


\section{UNIQUENESS OF MINIMAL MORPHISMS}

GS13 M. Gross and B. Siebert, Logarithmic Gromov-Witten invariants, J. Amer. Math. Soc. 26 (2013), no. 2, 451-510; doi:10.1090/S0894-0347-2012-00757-7.

Kat89 K. Kato, Logarithmic structures of Fontaine-Illusie, Algebraic Analysis, Geometry, and Number Theory (Baltimore, MD, 1988) (Johns Hopkins Univ. Press, Baltimore, MD, 1989), 191224.

SGA4-3 M. Artin A. Grothendieck, and J.-L. Verdier, Séminaire de Géométrie Algébrique du Bois-Marie 1963-1964 (SGA 4), Vol. 3, Théorie des topos et cohomologie étale des schémas, Lecture Notes in Math., vol. 305 (Springer-Verlag, Berlin - New York, 1973); doi:10.1007/BFb0070714.

Wis16 J. Wise, Moduli of morphisms of logarithmic schemes, Algebra Number Theory 10 (2016), no. 4, 695-735; doi:10.2140/ant.2016.10.695.

Jonathan Wise jonathan.wise@colorado.edu

University of Colorado, Campus Box 395, Boulder, CO 80309-0395, USA 